\title{
Photodynamic therapy for COVID-19
}

To the Editor - The case count of COVID-19 continues to grow since its emergence in late December 2019, with more than 23.7 million cases globally. A variety of pharmaceutical therapies are under investigation, including therapies that block viral binding (that is, monoclonal antibodies, recombinant angiotensin-converting enzyme 2 (ACE-2), transmembrane protease, serine 2

(TMPRSS2) inhibitors, and so on), inhibit viral RNA production (that is, antivirals), block the release of mature virion particles (that is, hydroxychloroquine), modulate pro-inflammatory cytokines (that is, anti-interleukin-6 agents, glucocorticoids), attentuate the clotting effects of the host response (that is, anticoagulants), or support the immune system response (that is, immunoglobulins or convalescent plasma $)^{1,2}$. To date, the only drug therapies that have been approved for emergency use authorization (EAU) in the US and that have shown early moderate to significant signs of effectiveness are remdesivir and dexamethasone, respectively ${ }^{3,4}$. Moreover, some of these drugs, whether EAU, off-label or investigational, may pose serious side effects in patients who are already clinically deteriorating. Beyond investigational therapies, established intensive care unit protocols typically used to treat acute respiratory distress syndrome (ARDS) are failing in patients with severe COVID-195. ARDS is typically described as a state in which fluid build up in the alveoli of the lung inhibits sufficient oxygen exchange. Current supportive care of COVID-19-induced ARDS include steroids, interleukin-6 inhibitors, non-invasive oxygen therapy (high-flow nasal cannula), or invasive oxygen therapy (mechanical ventilation). Despite the many treatment options, none have proven overly successful. Here we briefly explore the unique features of COVID-19 ARDS and how these features warrant the exploration on the use of photodynamic therapy (PDT) to treat COVID-19.

Given the unique challenges of COVID-19-induced ARDS, several physician groups have proposed to rename ARDS to CARDS to indicate a possibly different mechanism of respiratory distress ${ }^{6}$. More specifically, virologists have begun to further study the pathogenesis of severe acute respiratory syndrome coronavirus 2 (SARS-CoV-2).
A study that performed a conserved domain analysis, homology modelling and molecular docking proposed that SARS-CoV-2 binds to the heme groups in haemoglobin, leading to severe hypoxia. Heme is composed of a ring-like organic compound known as a porphyrin to which an iron atom is attached. It is the iron atom that binds oxygen as the blood travels between the lungs and the tissues. It is hypothesized that several non-structural proteins (orf1ab, ORF10 and ORF3a) attack the $1 \beta$-chain of haemoglobin, which dissociates iron to form porphyrin. This leads to the disruption in the passage of haemoglobin carrying oxygen from alveoli to capillaries ${ }^{7}$. Although the study is in preprint and some have criticized the study design, SARS-CoV-2-mediated attack on haemoglobin could explain the lack of efficacy with intubation therapy ${ }^{8}$. The attack on haemoglobin leads to desaturation, which coupled with the proliferation of pro-inflammatory cytokines cause the alveoli to become filled with fluid, white blood cells, mucus and the detritus of destroyed lung cells. The attack on haemoglobin and the lung tissue can lead to multi-organ failure.

Given the distinct features of CARDS and its attack on haemoglobin and porphyrins, we suggest treating CARDS by the injection of porphyrin-based photosensitizers either systemically or locally into the lungs through the pulmonary artery using micro-catheters. Initially, and without the need for photoactivation, the photosensitizer molecules may act as a 'decoy' in which the SARS-CoV-2 virions would attach to photosensitizer molecules instead of healthy lung tissue or attack healthy haemoglobin. This may improve clinical performance and increase oxygenation. However, to reduce viral load, illumination of the target lung tissue using a fibre-optic catheter to deliver low-power light of a characteristic absorption wavelength for the photosensitizer (typical range $450-800 \mathrm{~nm}$ ) causes photoactivation, yielding a highly reactive oxygen species capable of destroying the bonded SARS-CoV-2 virions to the photosensitizer molecules through peroxidation. The versatility of PDT has been clinically validated in a wide range of pathologies from oesophageal and certain lung cancers, to uses in treating age-related macular degeneration in the eye and in tumour-margin identification during brain cancer surgery. Additionally, it has been demonstrated that PDT can be used to destroy pathogenic microorganisms, such as bacteria, viruses, protozoa and fungi ${ }^{9-12}$. The predominant mechanism in PDT involves the generation of singlet oxygen $\left({ }^{1} \mathrm{O}_{2}\right)$. The diffusion distance of ${ }^{1} \mathrm{O}_{2}$ is around $0.01-0.02 \mu \mathrm{m}$ before being quenched, so the photosensitizers must be associated intimately with the target substrate for maximal impact ${ }^{13}$. Thus, the SARS-CoV-2 affinity for the heme structure of the photosensitizer predicts high viral destruction titers limited to the zone of photoactivation. The application of nanoparticle technology may provide opportunities to enhance delivery, bioavailability, selectivity and functionality of currently available photosensitizers while reducing side effects. In the absence of specialized photonics or in resource-limited settings, some PDTs may be activated using transthoracic continuous-wave ultrasound (sonodynamic therapy). In some clinical settings, there may be an incremental benefit in combining PDT and sonodynamic therapy. PDT does not preclude the use of conjunctive pharmacologic treatment of COVID-19, in fact, PDT combined with an existing therapy may prove the most effective approach. However, preclinical and clinical studies need to be performed to determine the feasibility, safety and efficacy of this approach.

\section{Nicholas Kipshidze (D) 1ه, Nicholas Yeo ${ }^{1,2}$ and Nodar Kipshidze (iD) 3 \\ ${ }^{1}$ NY Cardiovascular Research, LLC, New York, NY, USA. ${ }^{2}$ Veryan Medical Limited, Horsham, UK. ${ }^{3}$ NYU Langone Health, New York, NY, USA.

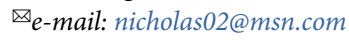

Published online: 2 October 2020 https://doi.org/10.1038/s41566-020-00703-9

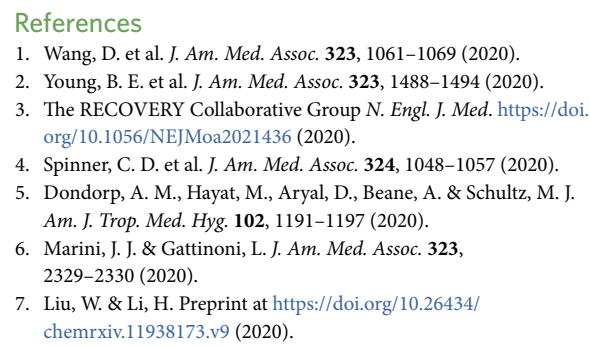


8. Read, R. J. Preprint at https://doi.org/10.26434/ chemrxiv.12120912.v1 (2020).

9. Kharkwal, G. B., Sharma, S. K., Huang, Y. Y., Dai, T. \& Hamblin, M. R. Lasers Surg. Med. 43, 755-767 (2011).

10. Majiya, H., Adeyemi, O. O., Herod, M., Stonehouse, N. J. \& Millner, P. J. Photochem. Photobiol. B 189, 87-94 (2018).

11. Wiehe, A., O'Brien, J. M. \& Senge, M. O. Photochem. Photobiol. Sci. 18, 2565-2612 (2019).
12. Caputo, N. D., Strayer, R. J. \& Levitan, R. Acad. Emerg. Med. 27, 375-378 (2020).

13. Moan, J. \& Berg, K. Photochem. Photobiol. 4, 549-553 (1991).

Acknowledgements

There is no funding source to report for this Correspondence.
Competing interests

The authors have the following unrelated industry relationships to disclose. Nicholas Kipshidze is a founder of and holds stock in Endobar Solutions LLC. Nodar Kipshidze is a consultant for Endobar Solutions LLC. N.Y. is an employee of Veryan Medical and a consultant for NY Cardiovascular Research. 